\title{
Inverse relation between evoked cortical association responses and behavioral orienting to repeated auditory stimuli
}

\author{
Jon A. Shaw and Richard F. Thompson \\ UNIVERSITY OF OREGON MEDICAL SCHOOL
}

\begin{abstract}
It is hypothesized that amplitudes of evoked cortical association responses will be inversely related to degree of "attention," the latter being operationally defined as the extent of behavioral orienting to a stimulus. Cats with implanted cortical recording electrodes were given series of clicks through either of two oppositely placed speakers. The degree and accuracy of the orienting response was rated, and evoked cortical association response simultaneously recorded, for each click. As predicted, the degree of the orienting response decreased significantly over trials, while the amplitude of the evoked association response exhibited a concomitant increase over trials.
\end{abstract}

\section{Problem}

Previous research indicated that evoked rèsponses recorded from cortical association areas of the waking cat are comparable in most characteristics to those obtained from the chloralosed cat (Shaw \& Thompson, 1964). Indirect evidence obtained from the latter preparation suggests that such responses may represent focalized but nonspecific cortical projections of the ascending reticular activating system (ARAS) (cf Thompson, et al., 1963). Numerous studies indicate that the ARAS may be activated during "attention" (cf Lindsley, 1958). If it is assumed that the ARAS can be driven by sudden peripheral stimuli to produce evoked cortical association responses only to the degree that it is not activated, then amplitudes of cortical association responses should be inversely related to degree of behavioral "attention."

Perhaps the only unambiguous operational definition of "attention" is in terms of the behaviorally defined orienting response. Consequently it may be predicted that degree of behavioral orienting and amplitude of evoked cortical association response elicited by a given stimulus will be inversely related. Since the behavioral orienting response typically decreases (i.e., habituates) to repeated stimulation, amplitudes of evoked cortical association responses should exhibit a concomitant increase over trials. The present experiment was designed to test this hypothesis.

\section{Method}

Bipolar electrodes recording across the cortex were implanted in the posterior middle suprasylvian association response area in five cats one week prior to the experiment. In the experiment, individual evoked responses to click stimulation were recorded photo- graphically and the amplitude (baseline to peak) of the positive component (surface positive to depth) measured.

A method for rating degree of behavioral orienting developed by Thompson \& Welker (1963) was used. Animals are placed in a small chamber within a soundproof room and a click delivered through either of two speakers (random sequence) located on opposite sides at equal distances from the chamber. The extent to which the animal turns his head toward the correct speaker is rated on a four point scale $(0=$ no response; $4=$ rapid and complete response). In the present experiment each of the five cats was given 10 trials (clicks) per day for four days. Clicks were presented only when the animal was facing midway between speakers, and were at least 2 min. apart. The orienting response to each click was rated and each evoked cortical association response to click recorded photographically.

\section{Results}

Figure 1 shows mean evoked association response amplitudes (in percent) and mean orientation scores, as a function of trials. The data were averaged over the five cats and four days. There is a clear inverse relation between degree of orienting and amplitude of evoked association responses over trials. Orienting scores were divided into two categories $(0,1$ vs 2,3 , and 4) and compared for three blocks of trials (1-3, 4-6 and 7-10) using chi-square. The decrease over trials is highly significant (chi-square $=40.68 ; \mathrm{df}=2 ; \mathrm{p}<.001$ ). Amplitudes of evoked association responses were converted to percentage scores for each animal and evaluated by analysis of variance. The increase in response amplitude over trials is highly significant $(F=2.94$; $\mathrm{df}=9$ and $156 ; \mathrm{p}<.01)$. The degree of inverse relationship between evoked responses and behavioral responses over trials is considerable, as indicated by a rank correlation coefficient of -0.7 . Consequently, as predicted, there is significant inverse relation between the neural and behavioral measures.

\section{Diseussion}

The results shown in Fig. 1 demonstrate clearly that amplitudes of evoked cortical association responses tend to be inversely related to degree of behavioral orienting, at least under the conditions of this experiment. It was postulated that evoked association response amplitudes would be inversely related to degree of "attention." Extent of the behavioral orienting response is by definition the behavioral measure of degree of 


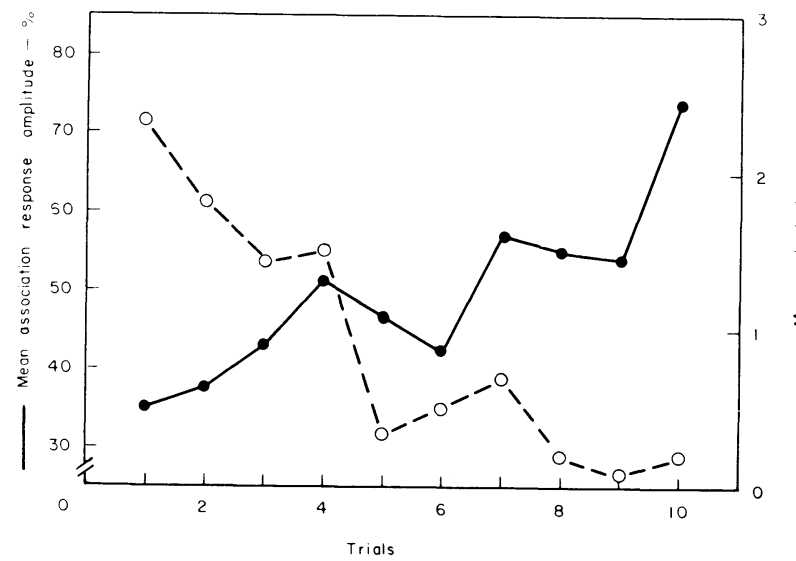

Fig. 1. Inverse relationship between mean evoked association response amplitudes (solid line and left ordinate) and mean orienting response scores (dotted line and right ordinate) over trials.

"attention." It is perhaps striking that these two independently defined neural and behavioral measures of "attention" should correspond so well. Results of previous experiments (Shaw \& Thompson, 1964) showing that cortical association response amplitudes to auditory, visual and tactile stimuli are markedly reduced by bodily activity and presentation of novel stimuli are consistent with this hypothesis.

Findings of the present study are in direct contrast to reports of habituation of primary evoked responses to repeated click stimulation (Hernandez-Peon, et al., 1957). Amplitudes of evoked cortical association responses increase rather than decrease, at least during the first few presentations of the stimulus. What does habituate is the concomitant behavioral orienting response. These findings are consistent with previous results indicating no long-term habituation of evoked association responses (Shaw \& Thompson, 1964). Once initial "attending" to the novel environment has habituated, evoked association responses should remain well developed and relatively constant. Hypothetical habituatory processes requiring hours of once every few second stimulation would seem to have no clear relation to behavioral phenomena.

\section{References}

HERNANDEZ-PEON, R., JOUVET, M., \& SCHERRER, H. Auditory potentials of cochlear nucleus during acoustic habituation. Acta Neurol. Latinoamer., 1957, 3, 144-156.

LINDSLEY, D. B. The reticular system and perceptual discrimination. In H. H. Jasper, (Ed.), Reticul a r Formation of the Brain. Boston: Little, Brown, 1958. Pp. 513-534.

SHAW, J. A., \& THOMPSON, R. F. Dependence of evoked cortical association responses on behavioral variables. Psychon. Sci., 1964, 1, 153-154.

THOMPSON, R. F., JOHNSON, R. H., \& HOOPES, J. J. Organization of auditory, somatic sensory, and visual projection to association fields of cerebral cortex in the cat. J. Neurophysiol., 1963, 26, 343-364.

THOMPSON, R. F., \& WELKER, W. I. Role of auditory cortex in reflex head orientation by cats to auditory stimuli. J. comp. physiol. Psychol., 1963, 56, 996-1002.

\section{Note}

1. Supported by grants from the National Institutes of Health (B-2161, NINDB; MH-K3-6650, NIMH) and the National Science Foundation (G-15603). 